\section{Evaluation the adjunctive use of combined bevacizumab and mitomycinc to trabeculectomy in management of recurrent pediatric glaucoma}

\author{
Abstract \\ Purpose To evaluate the efficacy and safety of \\ combined bevacizumab-mitomycin c (MMC) \\ in recurrent cases of pediatric glaucoma. \\ Methods A prospective non-masked \\ controlled study that included bilateral \\ cases of 12 patients (24 eyes) with recurrent \\ (had previous glaucoma surgery before) \\ pediatric glaucoma. One eye in each patient \\ (12 eyes) was assigned to trabeculectomy \\ operation with combined application of MMC \\ $(0.4 \mathrm{mg} / \mathrm{ml}$ for $3 \mathrm{~min})$ under and around the \\ scleral flap before trabeculectomy and \\ bevacizumab (avastin) (2.5 $\mathrm{mg}$ in $0.2 \mathrm{ml}$ ) \\ injected subconjunctivally around the bleb \\ after completing the surgery (group I). The \\ other eye of each patient (12 eyes) was assigned \\ to trabeculectomy operation with application of \\ MMC ( $0.4 \mathrm{mg} / \mathrm{ml}$ for $3 \mathrm{~min})$ only (group II). \\ The mean follow-up period was $13 \pm 1$ months. \\ Results The mean age was $2.16 \pm 1.5$ (range \\ 7 months to 4.1 years). No significant \\ difference in preoperative intraoperative \\ pressure (IOP) was observed between the \\ groups $(P>0.05)$. Recurrent primary \\ congenital glaucoma represents $66.7 \%$ of the \\ cases. Other cases included were recurrent \\ aphakic and pseudophakic glaucoma $25 \%$ and \\ recurrent post uveitic glaucoma $8.3 \%$. The \\ mean IOP was $12.1 \pm 4.2,12.6 \pm 5.4$, and \\ $12.8 \pm 5.2 \mathrm{~mm} \mathrm{Hg}$ in group $\mathrm{I}$ at 3,6 , and \\ 12 months, respectively, and was $12.8 \pm 5.3$, \\ $13.7 \pm 6.7$ and $15.6 \pm 5.9 \mathrm{~mm} \mathrm{Hg}$ in group II at \\ 3,6 , and 12 months, respectively. There was a \\ statistically significant difference in the mean \\ IOP between the studied groups at the 1-year
}

RA Mahdy, SM Al-Mosallamy, MA Al-Aswad, A Bor'i and WM El-Haig follow-up visit $(P<0.05)$. In addition, group I showed a higher statistically significant difference in absolute and total success (75 and $91.7 \%$, respectively) compared with group II (58.3 and 75\%, respectively) $(P<0.05)$. The encountered complications included mild hyphema, which occurred in $8.33 \%$ in group 1 , wound leakage, which occurred in $8.33 \%$ in each group, and shallow anterior chamber (AC), which occurred in $16.7 \%$ in each group and was the most common encountered complication in the study. One case of shallow AC in group I led to choroidal effusion $(8.33 \%)$. One case in group II developed late bleb-related endophthalmitis after 3 months, which resulted in phthisis bulbi $(8.33 \%)$.

Conclusion The additive effect of subconjunctival bevacizumab to MMCaugmented trabeculectomy in the case of recurrent pediatric glaucoma was beneficial in improving the success rate. Better IOP control and prolonging the bleb survivalvia reducing the long-term need of using anti-glaucoma drugs postoperatively without adding complications had also been achieved with this technique. This offers a promising alternative for the treatment of this type of glaucoma. Eye (2016) 30, 53-58; doi:10.1038/eye.2015.182; published online 2 October 2015

Introduction

Congenital glaucoma represents a challenging cause of blindness in children worldwide,
Faculty of Medicine,

Ophthalmology Department, Zagazig University, Sharkia, Egypt

Correspondence: RA Mahdy, Faculty of Medicine, Ophthalmology Department, Zagazig University, Sharkia 002, Egypt

Tel:+20 1155358466; Fax: +20 552384845 . E-mail: redaelmahdy2005@ yahoo.com

Received: 6 May 2015 Accepted in revised form: 4 August 2015 Published online: 2 October 2015 
despite its low incidence (1:10000 births). ${ }^{1}$ Its management is essentially surgical, with trabeculotomy and goniotomy serving as the first-choice procedures in most cases of primary congenital glaucoma. ${ }^{2,3}$ If intraocular pressure (IOP) is not effectively controlled by these procedures, then trabeculectomy is usually the next treatment option; however, variability exists in its success rate in these cases and ranges from 35 to $85 \%$, depending on the patient's age, number of previous surgeries, other pre-operative criteria, antimetabolite use, and length of follow-up period. ${ }^{4-7}$

Modulating the wound healing process to decrease scar formation around the scleral flap generally has a crucial role in the success rate of glaucoma filtering surgery. Antimetabolites, including mitomycin C (MMC) and 5-fluorouracil (5-FU), have been widely used with trabeculectomy to suppress the fibroblast proliferation. ${ }^{8}$ This is done by modulating the trans-differentiation of fibroblasts into myofibroblasts, which are chiefly responsible for scar formation, and hence increasing bleb survival with adequate IOP. ${ }^{9}$ Antimetabolites, such as 5-FU, can be given postoperatively or applied intraoperatively, as MMC. ${ }^{10}$ However, due to their nonspecific suppression effect on cell biology, their use may lead to cell damage, which can result in bleb leakage, hypotony, corneal scarring, and endophthalmitis. ${ }^{11}$

Vascular endothelial growth factor (VEGF) is a cytokine with multiple effects on wound healing processes through collagen deposition, angiogenesis, epithelialization, and scar formation. ${ }^{12}$ Bevacizumab (Avastin; Genentech, South San Francisco, CA, USA) is a recombinant humanized monoclonal immunoglobulin G1 antibody that directly inhibits VEGF. Bevacizumab was initially approved as a first-line treatment in metastatic colorectal cancer. ${ }^{13}$ In the field of ophthalmology, the promising results from clinical studies that utilized intravitreal bevacizumab injection to treat age-related macular degeneration, proliferative diabetic retinopathy, and iris neovascularization have led to trials involving different routes of bevacizumab administration to manage various eye disorders. ${ }^{14}$ Additionally, the topical or

subconjunctival application of bevacizumab was found to be valuable in inhibiting corneal neovascularization both in an experimental rat model and in humans. ${ }^{15,16}$ Better IOP control could be achieved with subconjunctival bevacizumab injection in glaucoma cases undergoing trabeculectomy. ${ }^{17}$ Experimentally bevacizumab not only enhances the beneficial effect of MMC on trabeculectomy outcome, but also allows reducing the toxicity of MMC via reducing its exposure time in mouse model. ${ }^{18}$

This study was conducted to evaluate the additive effect of intraoperative subconjunctival bevacizumab to MMC-augmented trabeculectomy in recurrent pediatric glaucoma concerning its efficacy, safety, and timing (shortly before glaucoma drainage devices or cyclodestructive procedures become mandatory).

\section{Materials and methods}

A prospective controlled study that included 12 patients of bilateral recurrent pediatric glaucoma. To participate in the study, all patients had an informed consent form signed by their parents. The protocol for the work has been approved by a suitably constituted Ethics Committee of the institution within which the work was undertaken and that it conforms to the provisions of the Declaration of Helsinki in 1995 (as revised in Edinburgh 2000).

The 12 patients represented 24 eyes that were selected from those attending the outpatient clinic of Zagazig University Hospital during the period from August 2012 to December 2012 and had undergone surgical management. One eye of each patient was treated by combined intraoperative bevacizumab and MMCaugmented trabeculectomy (group I included 12 eyes). The other eye of each patient was treated by trabeculectomy augmented with MMC only (group II included 12 eyes) and served as a control group. The time elapsed between treating the two eyes in the same patient was at least one week. Preoperative evaluation of the patients included the following demographic data: age, gender, family history, and follow-up duration. The details of the operation technique and the complications that occurred both during and after previous surgeries were also recorded. A complete ophthalmic history was taken, examination was done under general anesthesia before surgery including the measurement of IOP using a Perkins tonometer and the corneal diameter using caliper, anterior chamber (AC) depth and corneal clarity were assessed using the operating microscope. Fundus examination by direct and indirect ophthalmoscopy was performed depending on the clarity of the media.

\section{Inclusion criteria}

Patients with bilateral pediatric glaucoma (age $<12$ years) that has recurred after at least one previous surgery of trabeculectomy with or without MMC were included in the study.

\section{Exclusion criteria}

Patients with no perception of light or who had previous cyclodestructive procedures or glaucoma drainage devices were excluded. 


\section{Surgical technique}

All surgeries were performed under general anesthesia. Briefly, after a superior limbal-based conjunctival flap was prepared, a $2 \times 3 \mathrm{~mm}$ rectangular scleral flap of onethird to one-half of the scleral thickness was dissected toward the clear cornea. A solution of $0.4 \mathrm{mg} / \mathrm{ml} \mathrm{MMC}$ (Mitomycin C Kyowa manufactured by Biochem Pharmaceutical Inc., Mumbai, India) was prepared, and a $2 \times 4 \mathrm{~mm}$ surgical sponge soaked in the MMC solution was held in contact under the bed of scleral flap and extended to the area under the conjunctiva and Tenon. The conjunctiva was stretched over the sponge and held in place for $3 \mathrm{~min}$. Subsequently, the sponge was removed, and the entire area was carefully and continuously irrigated with $50 \mathrm{ml}$ of balanced saline solution (BSS) such that the falling fluid did not come in contact with the cornea. The procedure was then completed as a standard trabeculectomy.

A paracentesis was performed using number 15 keratome, which has the benefit of testing the filtration intraoperatively and aids in the reformation of the $\mathrm{AC}$ if it became lost postoperatively. Trabeculectomy was also performed using a number 15 keratome. Iridectomy was performed to prevent the risk of internal blockage of the trabeculectomy ostium. The scleral flap was sutured using a 10-0 nylon suture. The conjunctival/Tenon layer was sutured using a 10-0 nylon suture. Upon the completion of surgery in group I $(2.5 \mathrm{mg}$ in $0.2 \mathrm{ml}$ ) bevacizumab (Avastin; Genentech, San Francisco, CA, USA) was injected subconjunctivally using 27 gauge needle syringe on the three sides of the scleral flap to allow coverage of all subconjunctival area around the flap. In group II, sham injections were done. Topical cycloplegics, steroids, and antibiotics were administered postoperatively. Other than digital massage, no manipulations (such as bleb revision or suture-lysis) were performed unless they were necessary. Anti-glaucoma topical medications were used if IOP exceeded normal limits.

\section{Follow-up}

Cases were followed up at 1 day, 3 days, 1 week, 1 month, and every subsequent month for 12 months postoperatively. During each visit, ophthalmic examination was done under general anesthesias that performed preoperatively. Children of 3 years of age and older were sedated with chloral hydrate syrup (25-50 mg/kg body weight). Because some of the patients had some degree of corneal opacity that prevented the adequate evaluation of the optic disc, only intraocular pressure and any unexpected complications were analyzed.

Absolute success was defined as IOP equal to or less than $21 \mathrm{~mm} \mathrm{Hg}$ and equal to or greater than $5 \mathrm{~mm} \mathrm{Hg}$ without anti-glaucoma medications, without additional glaucoma surgery, without visually devastating complications, such as endophthalmitis or phthisis bulbi, and with no loss of light perception. Relative success was achieved when the above criteria were fulfilled but with additions of anti-glaucoma topical medical treatment.

\section{Statistical analysis}

Data were collected, tabulated, and statistically analyzed for the detection of significant values. Microsoft Excel 2007 was used to calculate the mean $\pm \mathrm{SD}$, Student's $t$-test was used to compare means, and Fisher's exact test was used to compare the percentage values. $P>0.05$ was considered as non-significant, $<0.05=$ significant,$<0.01=$ highly significant, and $<0.001=$ very highly significant.

\section{Results}

The demographic characteristics of the study groups are presented in Table 1. The mean age was $2.16 \pm 1.5$ (range 7 months to 4.1 years). There were no significant differences in preoperative IOP between the groups $(P>0.05)$. All patients in each group were diagnosed with advanced pediatric glaucoma with their clinical types, as shown in Table 1. The most common diagnosis was recurrent primary congenital glaucoma (16 eyes, 66.7\%). Other cases included were recurrent aphakic and pseudophakic glaucoma $25 \%$ and recurrent post uveitic glaucoma $8.3 \%$.

The mean IOP was $12.1 \pm 4.2,12.6 \pm 5.4$, and $12.8 \pm 5.2 \mathrm{~mm} \mathrm{Hg}$ in group I at 3, 6, and 12 months, respectively, and was $12.8 \pm 5.3,13.7 \pm 6.7$, and

Table 1 Patient demographics and preoperative characteristics

\begin{tabular}{|c|c|c|c|c|c|c|}
\hline \multirow[t]{2}{*}{ Item } & \multirow[t]{2}{*}{ Group I ( $\mathrm{n}=12$ eyes $)$} & \multirow[t]{2}{*}{ Group II ( $\mathrm{n}=12$ eyes) } & \multicolumn{2}{|c|}{ Total } & \multirow[t]{2}{*}{ T-test } & \multirow[t]{2}{*}{ P-value } \\
\hline & & & No. & $\%$ & & \\
\hline Age: mean $\pm S D$, Range & \multicolumn{5}{|c|}{$2.16 \pm 1.5,7$ months to 4.1 years (the cases were bilateral) } & \\
\hline \multicolumn{7}{|l|}{ Preoperative diagnosis } \\
\hline Primary Congenital glaucoma & 8 & 8 & 16 & 66.7 & 0.002 & $>0.05$ \\
\hline Aphakic and pseudo-aphakic glaucoma & 3 & 3 & 6 & 25 & 0.003 & $>0.05$ \\
\hline Post uvitic glaucoma & 1 & 1 & 2 & 8.3 & 0.004 & $>0.05$ \\
\hline Preoperative IOP & $29.2 \pm 3.6$ & $31.5 \pm 2.7$ & & & & 0.091 \\
\hline
\end{tabular}


Table 2 Mean IOP values at pre- and postoperative visits of both groups

\begin{tabular}{lccccc}
\hline Groups & Preop. & Day 1 & 1 month & 3 months & 1 year \\
\hline Group I & $29.2 \pm 3.6$ & $8.7 \pm 2.3$ & $11.6 \pm 3.8$ & $12.1 \pm 4.2$ & $12.6 \pm 5.4$ \\
Group II & $31.5 \pm 2.7$ & $9.1 \pm 1.5$ & $10.9 \pm 4.5$ & $12.8 \pm 5.3$ & $13.7 \pm 6.7$ \\
T-test & 0.0344 & 0.0411 & 0.0752 & 0.0779 & 0.1092 \\
P-value & 0.091 & 0.0823 & 0.0795 & 0.077 & 0.0510 \\
\hline
\end{tabular}

* Statistically significant showed statistically significant difference in IOP between the studied groups at only the last postoperative visit $(P<0.05)$.

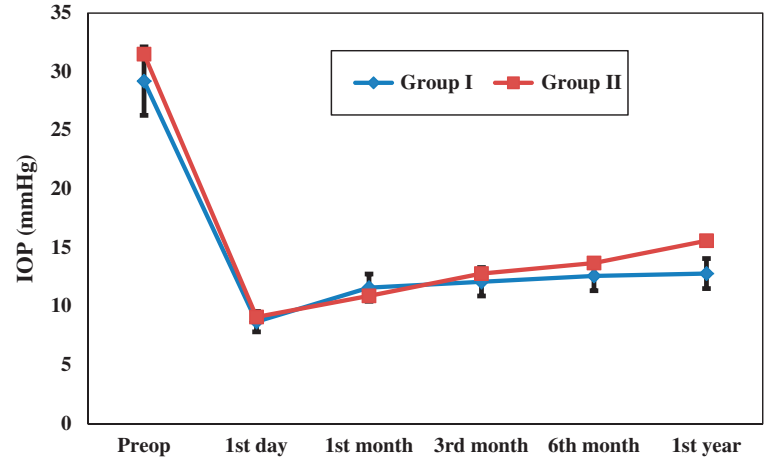

Figure 1 Linear regression curve showing preoperative and postoperative follow-up of the IOP with error bar.

$15.6 \pm 5.9 \mathrm{~mm} \mathrm{Hg}$ in group II at 3, 6, and 12 months, respectively. There was a statistically significant difference in the mean IOP between the studied groups at the 1-year follow-up visit $(P<0.05)$ (Table 2; and as shown in Figure 1). In addition, group I showed a higher statistically significant difference in absolute and total success (75 and 91.7\%, respectively) compared with group II (58.3 and $75 \%$, respectively) $(P<0.05)$ (Table 3).

The operative complication of hyphema occurred in one case in group $1(8.33 \%)$ and was mild and resolved spontaneously within the first two postoperative days. Conjunctival wound leak occurred in one eye $(8.33 \%)$ in each group, which required re-suturing before the patients were discharged from the hospital. Shallow AC occurred in two cases $(16.7 \%)$ in each group, three of these cases reformed spontaneously within 3-5 days postoperatively and were not sufficiently severe to require surgical interventions.

One case $(8.33 \%)$ of shallow $\mathrm{AC}$ in group I led to choroidal effusion and resolved after surgical reformation of AC. One case $(8.33 \%)$ in group II developed late-onset endophthalmitis after the 3-month follow-up; this was a bleb-related infection that was managed by systemic and topical fortified antibiotic eye drops according to the culture and sensitivity of the aqueous and vitreous samples. Additionally, intravitreal and subconjunctival injections were given, but unfortunately, the patient
Table 3 Absolute and relative success of both groups

\begin{tabular}{|c|c|c|c|c|c|c|}
\hline & \multicolumn{2}{|c|}{$\begin{array}{c}\text { Group } 1 \\
(\mathrm{~N}=12 \\
\text { eyes })\end{array}$} & \multicolumn{2}{|c|}{$\begin{array}{c}\text { Group } 2 \\
(\mathrm{~N}=12 \\
\text { eyes })\end{array}$} & \multirow[t]{2}{*}{ F-value } & \multirow[t]{2}{*}{ P-value } \\
\hline & No. & $\%$ & No. & $\%$ & & \\
\hline Absolute success & 9 & 75 & 7 & 58.3 & 0.443 & $0.0455^{*}$ \\
\hline Relative success & 2 & 16.7 & 2 & 16.7 & 0.138 & 0.0521 \\
\hline Total success & 11 & 91.7 & 9 & 75 & 0.612 & $0.0386^{*}$ \\
\hline
\end{tabular}

${ }^{*}$ Group I showed statistically significant difference in absolute and total success compared with group II $(P>0.05)$.

Table 4 Complications encountered in the two study groups

\begin{tabular}{|c|c|c|c|c|c|c|}
\hline \multirow[t]{2}{*}{ Complications } & \multicolumn{2}{|c|}{$\begin{array}{l}\text { Group I, } \\
\mathrm{N}=12\end{array}$} & \multicolumn{2}{|c|}{$\begin{array}{l}\text { Group II, } \\
\mathrm{N}=12\end{array}$} & \multicolumn{2}{|c|}{ Total } \\
\hline & No. & $\%$ & No. & $\%$ & No. & $\%$ \\
\hline Hyphema & 1 & 8.33 & - & & 1 & 8.33 \\
\hline Wound leakage & 1 & 8.33 & 1 & 8.33 & 2 & 16.7 \\
\hline Shallow anterior chamber & 2 & 16.7 & 2 & 16.7 & 4 & 33.3 \\
\hline $\begin{array}{l}\text { Serous choroidal } \\
\text { detachment }\end{array}$ & 1 & 8.33 & - & 8.33 & 1 & 8.33 \\
\hline Endophthalmitis & - & & 1 & & 1 & 8.33 \\
\hline
\end{tabular}

experienced loss of light perception and ended in phthisis bulbi (Table 4).

\section{Discussion}

In pediatric glaucoma, it has been reported that trabeculectomy without adjunctives achieves only 30$50 \%$ success rates. ${ }^{7} \mathrm{MMC}$ is a potent suppressor of fibroblast proliferation when used intraoperatively, especially in cases in which previous surgery has failed, and has a greater rate of success and induces a greater reduction of IOP. ${ }^{19,20}$

Angiogenesis is a process of new blood vessel formation and is considered as a key element in the proliferative phase of wound healing and scar tissue formation. VEGF inhibition not only reduced the amount of scar formed but also improved the quality of the scar tissue by shifting the collagen fibril distribution in a manner that is similar to that of normal tissue. ${ }^{21}$ 
The subconjunctival injection of bevacizumab was found to be associated with improvements in trabeculectomy bleb survival in glaucoma, which suggests that bevacizumab may be a useful agent for improving the success rate and limiting scar tissue formation after trabeculectomy. ${ }^{22}$

This study aimed to evaluate the additive effect of bevacizumab to MMC-augmented trabeculectomy in recurrent cases of childhood glaucoma concerning its efficacy and complications. Our results revealed that the mean age was $2.16 \pm 1.5$ (range 7 months to 4.1 years); this explains the failure of the primary surgery in these children because a younger age results in a lower success rate. No significant differences in preoperative IOP were observed between the groups $(P>0.05)$.

All patients in each group suffered from advanced pediatric glaucoma, primarily recurrent primary congenital glaucoma, which represented $66.7 \%$ of the cases. Other cases included were recurrent aphakic and pseudophakic glaucoma $25 \%$ and recurrent post uveitic glaucoma $8.3 \%$. The two beneficial effects of adding bevacizumab to MMC trabeculectomy were the following: first, the postoperative IOP was lower in the first group, and this difference became statistically significant in the last postoperative (at 1 year) follow-up visit $(P<0.05)$. Second, group I showed a higher statistically significant difference in absolute and total success compared with group II $(P<0.05)$, which can be explained by the fact that less scar tissue was formed when combined bevacizumab-MMC, as opposed to only MMC, was used; this resulted in the improvement of the survival of the trabeculectomy operation.

The encountered complications included mild hyphema, which occurred in one eye in group 1 (8.33\%), wound leak which occurred in one eye $(8.33 \%)$ in each group and shallow AC, which occurred in two cases $(16.7 \%)$ in each group and was the most common encountered complication in our study. One case $(8.33 \%)$ of shallow AC in group I led to choroidal effusion. One case $(8.33 \%)$ in group II developed late bleb-related endophthalmitis after 3 months, which ended in phthisis bulbi. The complication rate was comparable between the groups.

In a long-term study conducted by Giampani et al, ${ }^{23}$ they found that MMC-augmented trabeculectomy was successful in $55.26 \%$ within $61.16 \pm 26.13$ months of follow-up for childhood glaucoma. This lower success rate compared with our study $(75 \%)$ can be attributed to the longer follow-up period than that used in our study. In that study, they also found that endophthalmitis appeared in eight eyes $(4.88 \%)$, which was comparable to our study ( 1 of 24 eyes $=4.16 \%$ ).
Chen et $\mathrm{l}^{24}$ reported a success rate of $67 \%$ for trabeculectomy with MMC in primary congenital glaucoma with a concentration of $0.2 \mathrm{mg} / \mathrm{ml}$ for $3 \mathrm{~min}$.

In a case series study conducted by Choi et al, ${ }^{25}$ they used combined subconjunctival bevacizumab as an adjunct to MMC trabeculectomy (as in our study) in eyes with refractory glaucoma but did not include pediatric cases. The results suggest that subconjunctival bevacizumab administration may be an effective and safe adjunct regimen to trabeculectomy in eyes with refractory glaucoma because the blockage of angiogenesis and possible fibroblast modulation with anti-VEGF agent may provide benefits for glaucoma filtering surgery.

Biteli and Prata ${ }^{26}$ used subconjunctival bevacizumab as an adjuvant therapy in first-time MMC augmented filtration surgery for patients with primary glaucoma; the complete success rates at 12 months ranged between 71 and $88 \%$, and the qualified success rates ranged between 84 and $96 \%$. This study reported a higher success rate than our study because their cases were primarily non-pediatric, non-recurrent glaucoma cases. Recurrent cases were included in a study performed by Saeed and AboulNasr. ${ }^{27}$ At the end of the 24-month follow-up, they found a cumulative probability of 0.769 and 0.231 for complete and qualified success, respectively, in the combined avastin-MMC trabeculectomy group. In comparison, they found cumulative probabilities of 0.538 and 0.308 for complete and qualified success, respectively, in MMC trabeculectomy. However, the authors excluded the congenital glaucoma cases from their study.

To the best of our knowledge, no previous studies have used the combined subconjunctival bevacizumab as an adjunct to MMC trabeculectomy in recurrent childhood glaucoma.

\section{Conclusion}

The additive effect of subconjunctival bevacizumab to MMC-augmented trabeculectomy in cases of recurrent childhood glaucoma was beneficial in improving the success rate with better IOP control and prolonging the trabeculectomy survivalvia reducing the long-term need of using anti-glaucoma drugs postoperatively without adding complications. This offers a promising alternative for the treatment of this type of glaucoma. However, before reaching a final conclusion and establishing this treatment strategy, it is necessary to conduct the same study protocol in a large multicenter study with a longer follow-up period. 


\section{Summary}

What was known before

- The additive effect of subconjunctival bevacizumab to MMC augmented trabeculectomy in the case of recurrent pediatric glaucoma was beneficial in improving the success with better IOP control and prolonging the bleb survival without adding complications.

What this study adds

- This offers a promising alternative for the treatment of this type of glaucoma.

\section{Conflict of interest}

The authors declare no conflict of interest.

\section{Acknowledgements}

The protocol for the work has been approved by a suitably constituted Ethics Committee of the institution within which the work was undertaken and that it conforms to the provisions of the Declaration of Helsinki in 1995 (as revised in Edinburgh 2000). The work has not previously been presented at a meeting.

\section{References}

1 Dickens CJ, Hoskins HD. Diagnosis and treatment of congenital glaucoma. In: Ritch R, Shields MB, Krupin T (eds). The Glaucomas. Mosby, St. Louis, 1989.

2 Anderson DR. Trabeculotomy compared to goniotomy for glaucoma in children. Ophthalmology 1983; 90: 805-806.

3 Luntz MH, Livingston DG. Trabeculotomy-ab-externo and trab-eculectomy in congenital and adult onset glaucoma. Am J Ophthalmol 1977; 83: 174-179.

4 Beauchamp GR, Parks MM. Filtering surgery in children: barriers to success. Ophthalmology 1979; 86: 170-180.

5 Inaba Z. Long-term results of trabeculectomy in the Japanese: an analysis by life-table method. Jpn J Ophthalmol 1982; 26: 361-373.

6 Gressel MG, Heuer DK, Parrish RK. Trabeculectomy in young patients. Ophthalmology 1984; 91: 1242-1246.

7 Debnath SC, Teichmann KD, Salamah K. Trabeculectomy versus trabeculotomy in congenital glaucoma. $\mathrm{Br} J$ Ophthalmol 1989; 73: 608-611.

8 Lama PJ, Fechtner RD. Antifibrotics and wound healing in glaucoma surgery. Surv Ophthalmol 2003; 48: 314-346.

9 Reddick R, Merritt JC, Ross G, Avery A, Peiffer RL. Myofibroblasts in filtration operations. Ann Ophthalmol 1985; 17(3): 200-203.

10 Mostafa A. Augmenting trabecu-lectomy in glaucoma with subconjunctivalmitomycin $C$ versus subconjunctival 5-fluorouracil: a randomized clinical trial. Clin Ophthalmol 2011; 5: 491-494.

11 Seah SK, Prata Jr JA, Minckler DS, Baerveldt G, Lee PP, Heuer DK. Hypotony following trabeculectomy. J Glaucoma 1995; 4(2): 73-79.
12 Bao P, Kodra A, Tomic-Canic M, Golinko MS, Ehrlich HP, Brem H. The role of vascular endothelial growth factor in wound healing. J Surg Res. 2009; 153(2): 347-358.

13 Miller KD, Chap LI, Holmes FA, Cobleigh MA, Marcom PK, Fehrenbacher L et al. Randomized phase III trial of capecitabine compared with bevacizumab plus capecitabine in patients with previously treated metastatic breast cancer. J Clin Oncol 2005; 23: 792-799.

14 Wakabayashi T, Oshima Y, Sakaguchi H, Ikuno Y, Miki A, Gomi $\mathrm{F}$ et al. Intra-vitreal bevacizumab to treat iris neovascularization and neovascular glaucoma secondary to ischemic retinal diseases in 41 consecutive cases. Ophthalmology 2008; 115: 1571-1580.

15 Manzano RP, Peyman GA, Khan P, Carvounis PE, Kivilcim M, Ren $\mathrm{M}$ et al. Inhibition of experimental corneal neovascularisation by bevacizumab (Avastin). $\mathrm{Br} \mathrm{J}$ Ophthalmol 2007; 91: 804-807.

16 Kim SW, Ha BJ, Kim EK, Tchah H, Kim TI. The effect of topical bevacizumab on corneal neovascularization. Ophthalmology 2008; 115: e33-e38.

17 Grewal DS, Jain R, Kumar H, Grewal SP. Evaluation of subconjunctival bevacizumab as an adjunct to trabecul-ectomy: a pilot study. Ophthalmology 2008; 115: 2141-2145.

18 Van Bergen T, Vandewalle E, Moons L, Stalmans. Complementary effects of bevacizumab and MMC in the improvement of surgical outcome after glaucoma filtration surgery. Acta Ophthalmol 2015; e-pub ahead of print 18 May 2015; doi:10.1111/aos.12766.

19 Lamping KA, Belkin JK. 5-Fluorouracil and Mitomycin C in pseudophakic patients. Ophthalmology 1995; 102: 70-75.

20 Higginbotham EJ, Stevens RK, Musch DC. Bleb-related endophthalmitis after trabeculectomy with mitomycin C. Ophthalmology 1996; 103: 650-656.

21 Chaudhary NI, Roth GJ, Hilberg F, Müller-Quernheim J, Prasse A, Zissel G et al. Inhibition of PDGF, VEGF and FGF signalling attenuates fibrosis. Eur Respir J 2007; 29: 976-985.

22 Memarzadeh F, Varma R, Lin LT, Parikh JG, Dustin L, Alcaraz A et al. Postoperative use of bevacizumab as an antifibrotic agent in glaucoma filtration surgery in the rabbit. Invest Ophthalmol Vis Sci 2009; 50: 3233-3237.

23 Giampani Jr J, Borges-Giampani AS, Carani JC, Oltrogge EW, Susanna Jr R. Efficacy and safety of trabeculectomy with mitomycin C for childhood glaucoma: a study of results with long-term follow-up. Clinics 2008; 63(4): 421-426.

24 Chen Q, Yu Y, Sun H, Zong Y, Yuan ZL. A retrospective and consecutive study of viscocanalostomy versus trabeculectomy for primary congenital glaucoma. Chin Med J 2013; 126(8): 1418-1424.

25 Choi JY, Choi J, Kim Y-D. Subconjunctival bevacizumab as an adjunct to trabeculectomy in eyes with refractory glaucoma: a case series. Korean J Ophthalmol 2010; 24(1): $47-52$.

26 Biteli LG, Prata TS. Subconjunctival bevacizumab as an adjuvant in first-time filtration surgery for patients with primary glaucomas. Int Ophthalmol 2013; 33(6): 741-746.

27 Saeed AM, AboulNasr TT. Subconjunctival bevacizumab to augment trabeculectomy with mitomycin $\mathrm{C}$ in the management of failed glaucoma surgery. Clin Ophthalmol 2014; 8: 1745-1755. 